\title{
Comparison of the effectiveness of using the optic strut and tuberculum sellae as radiological landmarks in diagnosing paraclinoid aneurysms with CT angiography
}

\author{
Chih-Hsiang Liao, MD, ${ }^{1}$ Chung-Jung Lin, MD, ${ }^{2,3}$ Chun-Fu Lin, MD, ${ }^{1,3}$ Hsin-Yi Huang, MS, ${ }^{4}$ \\ Min-Hsiung Chen, MD, ${ }^{1,3}$ Sanford P. C. Hsu, MD, ${ }^{1,3}$ and Yang-Hsin Shih, MD ${ }^{1,3}$
}

Departments of ${ }^{1}$ Neurosurgery, Neurological Institute, and ${ }^{2}$ Radiology and ${ }^{4}$ Biostatistics Task Force (under Department of Medical Research and Education), Taipei Veterans General Hospital; and ${ }^{3}$ National Yang Ming University, School of Medicine, Taipei, Taiwan, Republic of China

\begin{abstract}
OBJECTIVE The treatment of paraclinoid aneurysms remains challenging. It is important to determine the exact location of the paraclinoid aneurysm when considering treatment options. The authors herein evaluated the effectiveness of using the optic strut (OS) and tuberculum sellae (TS) as radiographic landmarks for distinguishing between intradural and extradural paraclinoid aneurysms on source images from CT angiography (CTA).

METHODS Between January 2010 and September 2013, a total of 49 surgical patients with the preoperative diagnoses of paraclinoid aneurysm and 1 symptomatic cavernous-clinoid aneurysm were retrospectively identified. With the source images from CTA, the OS and the TS were used as landmarks to predict the location of the paraclinoid aneurysm and its relation to the distal dural ring (DDR). The operative findings were examined to confirm the definitive location of the paraclinoid aneurysm. Statistical analysis was performed to determine the diagnostic effectiveness of the landmarks.
\end{abstract}

RESULTS Nineteen patients without preoperative CTA were excluded. The remaining 30 patients comprised the current study. The intraoperative findings confirmed 12 intradural, 12 transitional, and 6 extradural paraclinoid aneurysms, the diagnoses of which were significantly related to the type of aneurysm $(p<0.05)$ but not factors like sex, age, laterality of aneurysm, or relation of the aneurysm to the ophthalmic artery on digital subtraction angiography. To measure agreement with the correct diagnosis, the OS as a reference point was far superior to the TS (Cohen's kappa coefficients 0.462 and 0.138 for the OS and the TS, respectively). For paraclinoid aneurysms of the medial or posterior type, using the base of the OS as a reference point tended to overestimate intradural paraclinoid aneurysms. The receiver operating characteristic curve indicated that if the aneurysmal neck traverses the axial plane $2 \mathrm{~mm}$ above the base of the OS, the aneurysm is most likely to grow across the DDR and present as a transitional aneurysm (sensitivity 0.806; specificity $0.792)$.

CONCLUSIONS High-resolution thin-cut CTA is a fast and crucial tool for diagnosing paraclinoid aneurysms. The OS serves as an effective landmark in CTA source images for distinguishing between intradural and extradural paraclinoid aneurysms. The DDR is supposed to be located $2 \mathrm{~mm}$ above the base of the OS in axial planes.

http://thejns.org/doi/abs/10.3171/2015.7.JNS151046

KEY WORDS computed tomographic angiography; distal dural ring; optic strut; paraclinoid aneurysm; proximal dural ring; tuberculum sellae; vascular disorders

$\mathrm{T}$ HE treatment of paraclinoid aneurysms remains challenging. $7,8,10,17,18,25$ It is important to differentiate the exact location of the paraclinoid aneurysm, whether intradural or not, when considering treatment options. The distal dural ring (DDR) is the anatomical boundary between the intra- and extradural segments of the internal carotid artery (ICA) in the paraclinoid region. ${ }^{2,9,19,24}$ Some studies tried to delineate the DDR with imaging methods and differentiate the aneurysm in the vicinity of the anterior clinoid process (ACP) ${ }^{5,16,23}$ However, these studies either lacked direct surgical confirmation or comprised a small case number.

ABBREVIATIONS $A C P=$ anterior clinoid process; $C_{4}=$ cavernous segment; $C_{5}=$ clinoid segment; $C_{6}=$ ophthalmic segment; $C T A=C T$ angiography; $D D R=$ distal dural ring; $\mathrm{DSA}=$ digital subtraction angiography; $\mathrm{ICA}=$ internal carotid artery; $\mathrm{OphA}=$ ophthalmic artery; $\mathrm{OS}=$ optic strut; $\mathrm{PDR}=$ proximal dural ring; $\mathrm{ROC}=$ receiver operating characteristic; TS = tuberculum sellae.

SUBMITTED May 6, 2015. ACCEPTED July 27, 2015.

INCLUDE WHEN CITING Published online January 8, 2016; DOI: 10.3171/2015.7.JNS151046. 
The ACP is a short triangular bony projection of the lesser sphenoid wing and attached to the body of the sphenoid by 1 superior root, the roof of the optic canal, and 1 inferior root, the optic strut (OS). The OS is also the anterior limit of the clinoid $\left(\mathrm{C}_{5}\right)$ segment of the ICA, and the proximal dural ring (PDR) attaches to the inferior margin of the OS and is the roof of the cavernous sinus. ${ }^{21}$ The DDR and PDR are separated by the OS and the bulk of the ACP anteriorly but are fused at the tip of the ACP posteriorly. In addition, the medial part of the DDR is continuous with the dura covering the tuberculum sellae (TS). In the current study, we used the OS and the TS as radiological landmarks in source images from CT angiography (CTA) to determine if the paraclinoid aneurysm was intra- or extradural (i.e., either distal or proximal to the DDR). The definitive diagnoses of the paraclinoid aneurysms were confirmed by direct surgical inspection. The diagnostic effectiveness of these 2 landmarks was analyzed, and the relationship between the DDR and landmark was defined through statistics.

\section{Methods}

\section{Patient Group and Selection Criteria}

Between January 2010 and September 2013, 49 consecutive surgical patients with the preoperative diagnosis of paraclinoid aneurysm, either ruptured or not, were recruited retrospectively from the operation logbook. The preoperative diagnoses were based on CTA and/or digital subtraction angiography (DSA). CTA was a prerequisite. Patients without preoperative CTA or operation videos were excluded. This retrospective study was approved by the institutional review board of our hospital.

\section{Study Design}

To determine if the paraclinoid aneurysm is intra- or extradural (i.e., either distal or proximal to the DDR), 2 bony landmarks, the $\mathrm{TS}^{19}$ and OS, were chosen as reference points (Fig. 1B and C). Aneurysms located below these reference points were regarded as extradural, and aneurysms above were regarded as intradural. In this retrospective study, we used the source images from the CTA and operation videos to analyze the diagnostic effectiveness of these 2 landmarks.

\section{CTA Protocol}

High-resolution CTA with a multidetector CT scanner (Brilliance 64; Philips Healthcare) was applied for paraclinoid aneurysms. 3D CTA scans were obtained with the following parameters: $120 \mathrm{kV}, 250 \mathrm{~mA}$, field of view 22 to $24 \mathrm{~cm}$, matrix size $512 \times 512$, and UB filter type. The reconstructed axial image was $1 \mathrm{~mm}$ thick with $0.625-\mathrm{mm}$ intervals.

\section{Measurement}

At a 3D workstation (Philips Brilliance Workspace CT workstation), the axial orientation was adjusted to an angle parallel to the planum sphenoidale. The base of the OS is defined as the midpoint along the slope of the greater sphenoid wing where the OS attaches on the coronal multiplanar reformats (Figs. 2 and 3). One neuroradiolo-
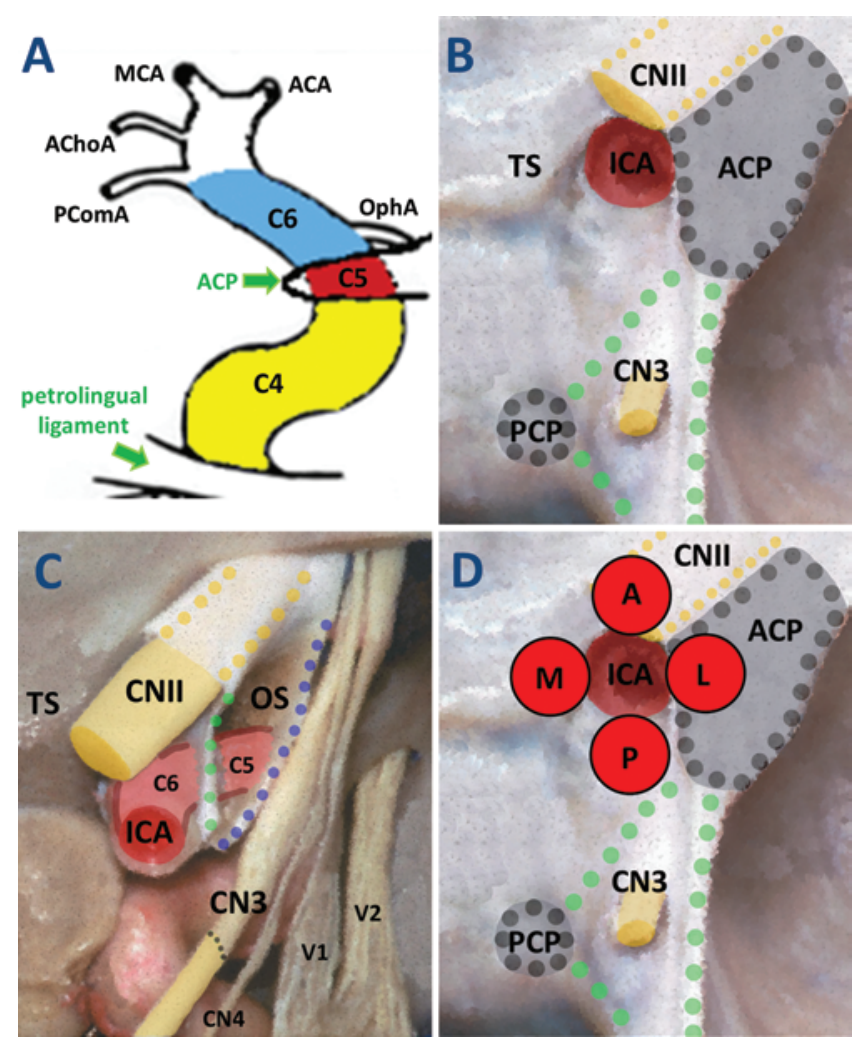

FIG. 1. ICA classification and the anatomy discussed in this study. A: The Bouthillier nomenclature for ICA segments. B: Superior view of the right paraclinoid region (dura preserved). The area surrounded by the green dots is the oculomotor triangle, where third cranial nerve (CN3) pierces into the cavernous sinus. C: Superior view of the right paraclinoid region (dura and ACP removed). The green dots represent the DDR, the purple dots represent the PDR, and the $\mathrm{C}_{5}$ segment of the ICA and OS is flanked by the rings. D: According to the neck origin of the paraclinoid aneurysm on the ICA, we classified the aneurysms as anterior $(A)$, medial $(M)$, lateral $(L)$, and posterior $(P)$ types. ACA = anterior cerebral artery; $\mathrm{AChoA}=$ anterior choroidal artery; $\mathrm{CNII}=$ optic nerve; $\mathrm{CN} 3$ = oculomotor nerve; $\mathrm{CN} 4$ = trochlear nerve; $\mathrm{MCA}=$ middle cerebral artery; $\mathrm{PComA}=$ posterior communicating artery; $\mathrm{PCP}$ $=$ posterior clinoid process; $V_{1}=$ ophthalmic nerve; $V_{2}=$ maxillary nerve . Panels $B-D$ are reproduced and used with permission from Rhoton $A L$ Jr: The cavernous sinus, the cavernous venous plexus, and the carotid collar. Neurosurgery 51 (4 Suppl):S375-S410, 2002. Figure is available in color online only.

gist (C.J.L.) and 1 neurosurgeon (C.H.L.), who were both blinded to the intraoperative findings and definitive diagnoses, concurred on the position of the base of the OS, which was simultaneously cross-referenced in the axial and sagittal planes. The same coronal cut used to define the base of the OS was also used to define the location of the TS in the axial and sagittal planes with the aid of a digital cross-referencing tool.

The distances between the axial cuts where the base of the OS and the aneurysmal neck (both the most proximal and distal points) were measured (Figs. 2 and 3). The base of the OS is set as 0 . For example, if the proximal point of the aneurysmal neck is $3 \mathrm{~mm}$ below the base of the OS $(-3 \mathrm{~mm})$, and the distal point is $5 \mathrm{~mm}$ above $(+5 \mathrm{~mm})$, this aneurysm is then regarded as a transitional paraclinoid aneurysm. If both the proximal and distal points of the aneurysmal neck are below the OS, the preoperative diagnosis 

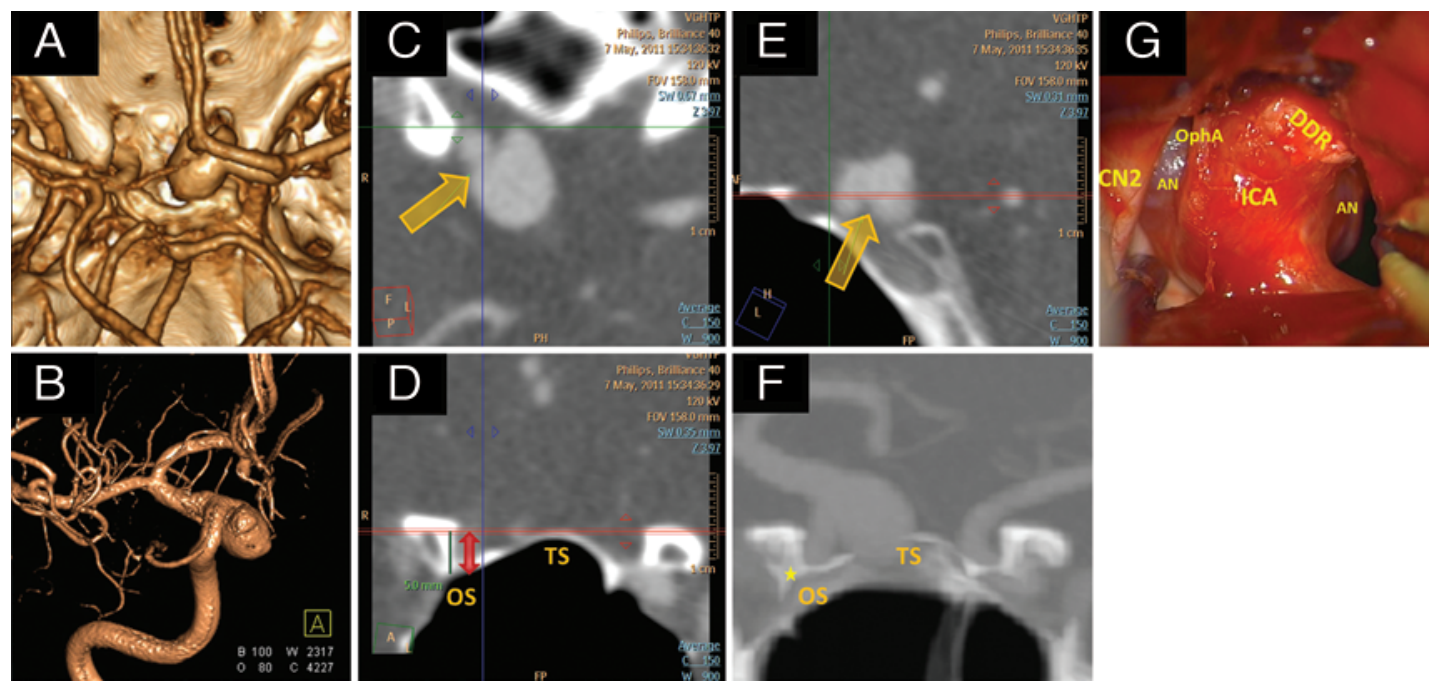

FIG. 2. Case 24. Measurement with the concordant diagnosis using both the OS and TS as the reference points. A and B: CT angiogram (A) and DS angiogram (B) showing a right paraclinoid aneurysm of the medial type. $\mathrm{C}$ and $\mathrm{E}$ : The proximal neck of the aneurysm is indicated by the yellow arrows on the axial $(C)$ and sagittal $(E)$ source CTA images. D: Using digital cross-referencing and measurement tools, the distances were determined ( $+5 \mathrm{~mm}$ for OS and $0 \mathrm{~mm}$ for TS). F: The usual reformatted 8-mm-thick coronal CTA image is shown for comparison and better delineation of the OS. The yellow star indicates the base of the OS. Detailed measurement was still performed on thin-cut source images with digital cross-referencing tools. The data of the distal neck were also retrieved (not shown here) in the same way $(+8.9 \mathrm{~mm}$ for OS and $+3.4 \mathrm{~mm}$ for TS). Hence, the preoperative diagnosis was an intradural paraclinoid aneurysm with both the OS and the TS as reference points. G: The pretemporal transclinoid/transcavernous approach along with extradural anterior clinoidectomy was used for clipping. The intraoperative findings confirmed that the diagnosis is correct. The aneurysm (AN) was located distal to the DDR. Figure is available in color online only.

is total extradural aneurysm; if both points were above the OS, the diagnosis is total intradural. Using the TS as another reference point, measurements were repeated with the methods identical to that of the OS.

In this study, the Bouthillier classification of ICA segments was adopted (Fig. 1A). ${ }^{1}$ We further classified paraclinoid aneurysms as the medial, lateral, anterior, or posterior type according to the neck origin of the aneurysm on the ICA for later discussion (Fig. 1D).

\section{Surgical Approach}

The pretemporal transclinoid/transcavernous approach along with extradural anterior clinoidectomy was used for clipping. ${ }^{12-15}$ The $\mathrm{C}_{5}$ segment of the ICA, DDR, and PDR were clearly exposed in the operation. The span of the aneurysmal neck and its relation with the rings were identified and confirmed.

\section{Statistical Analysis}

Each aneurysmal neck has the most proximal and distal points along the ICA. Hence, 2 sets of measurement data from each paraclinoid aneurysm could be retrieved to examine if the point of interest was intra- or extradural, the results of which were compared with the definitive intraoperative diagnoses using linear regression. To compare the diagnoses with other factors (sex, age, laterality of aneurysm, type of aneurysm, and relation of the aneurysm to the ophthalmic artery [OphA] on DSA), the chi-square test or McNemar's test for nominal variables and ANOVA for continuous variables were used. To measure agreement with the correct diagnosis (interrater reliability), Cohen's kappa coefficient was used. The Biostatistics Task Force of Taipei Veterans General Hospital assisted in the data processing and statistical analysis using IBM SPSS Statistics version 20.0.

\section{Results}

Of the 49 surgical patients with preoperative diagnoses of paraclinoid aneurysms, 19 patients without source images from preoperative CTA were excluded. The remaining 30 patients ( 7 men and 23 women) comprised the current retrospective study. The mean patient age was 56 years (range 37-76 years). The demographics, intraoperative diagnoses, and the measurement results of using the OS and TS as reference points in the CTA source images were shown in Table 1. There were 12 medial, 8 anterior, 2 lateral, and 8 posterior types of paraclinoid aneurysms in this study. The intraoperative findings confirmed 12 intradural, 12 transitional, and 6 extradural paraclinoid aneurysms. The follow-up DSA showed no residual aneurysms.

\section{Statistical Analysis}

The definitive intraoperative diagnoses were significantly related to the type of aneurysm, but not factors like sex, age, laterality of aneurysm, and relation of the aneurysm to the OphA on DSA (Table 2). Paraclinoid aneurysms of the medial type were mostly transitional or intradural, the reason for which was due to the carotid cave (discussed later). Paraclinoid aneurysms of the anterior type were either intradural or transitional. In this series, there were no extradural paraclinoid aneurysms of the anterior type, and there were no such aneurysms (i.e., anterior clinoid aneurysms) in our experience. We speculate that the anterior wall of the short extradural clinoid segment of the ICA does not take much hemodynamic force. 

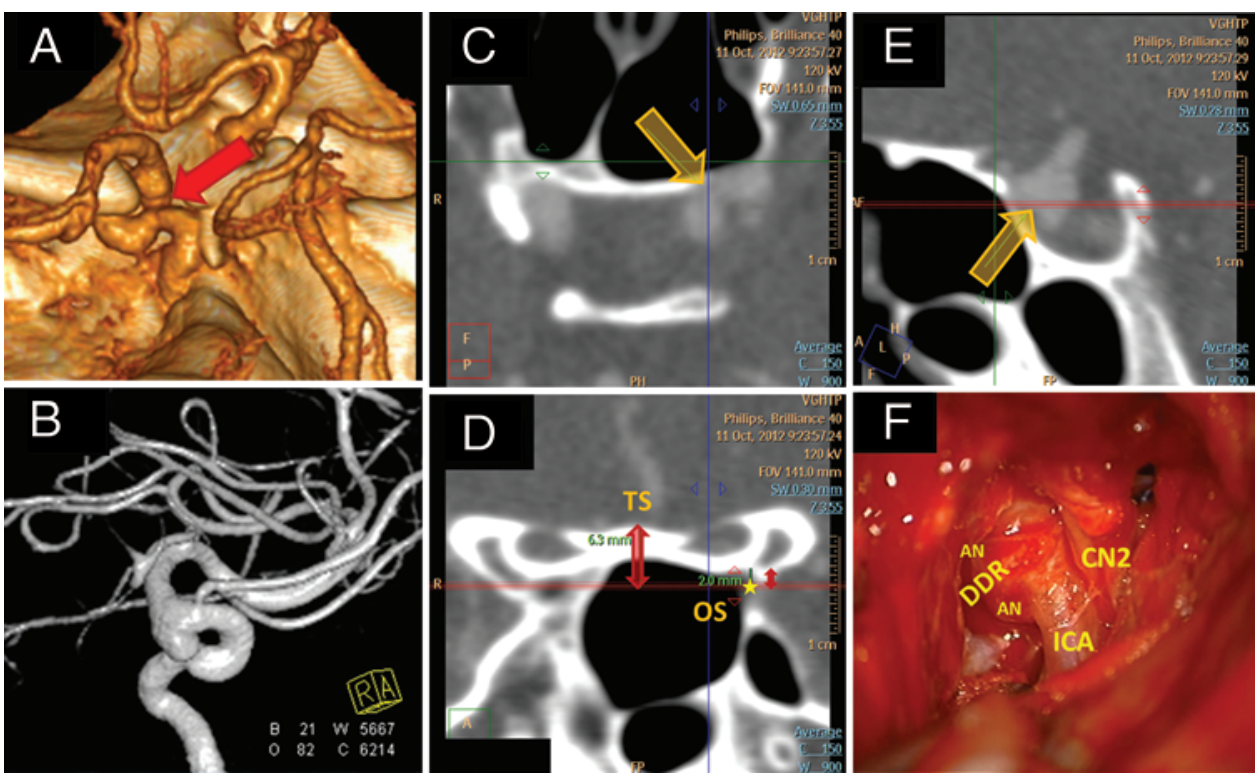

FIG. 3. Case 5. Measurement with concordant pre- and postoperative diagnosis using the OS as a reference point. A and B: CT angiogram $(A)$ and DS angiogram (B) showing a left paraclinoid aneurysm of the posterior type. $C$ and $E$ : The proximal neck of the aneurysm is indicated by the yellow arrows on axial (C) and sagittal (E) source CTA images. D: Using digital cross-referencing and measurement tools, the distances were determined $(-2.0 \mathrm{~mm}$ for OS and $-6.3 \mathrm{~mm}$ for TS). The yellow star stands the base of the OS. The data of the distal neck were also retrieved (not shown here) in the same way $(+3.0 \mathrm{~mm}$ for OS and $-1.9 \mathrm{~mm}$ for TS). Hence, the preoperative diagnosis was either a transitional paraclinoid aneurysm using the OS as a reference point or an extradural paraclinoid aneurysm using the TS as another reference point. F: The pretemporal transclinoid/transcavernous approach along with extradural anterior clinoidectomy was used for clipping. The intraoperative findings confirmed the aneurysm as transitional. The DDR spanned across the aneurysm and its neck. Figure is available in color online only.

Instead, the segment of the ICA between the DDR and the take-off site of the OphA takes most of the upward force. Paraclinoid aneurysms of the posterior type with a medial projection component tended to be transitional or intradural. The number of paraclinoid aneurysms of the lateral type was too small to analyze.

\section{Is the OS or TS Better?}

In this study, we found that the definitive diagnoses and the vertical distances between the axial planes of the aneurysmal neck and the reference points were significantly correlated in the linear regression (0.921 and 0.905 for the OS and TS, respectively). Comparing the diagnostic accuracy of the OS and TS, the OS is far superior to the TS ( $\kappa 0.462$ and 0.138 for the OS and TS, respectively), which means that the OS has a higher interrater reliability and serves as a better radiological landmark.

In addition, there were no correlations between the definitive diagnoses and the horizontal distances between the sagittal planes of the aneurysmal neck and each reference point.

\section{Medial Type, Carotid Cave, and Superior Hypophyseal Artery Aneurysms}

While the lateral aspect of the DDR is tightly adherent to the ICA, the medial aspect of it is attached more loosely and may be incomplete, allowing a small pouch of subarachnoid space to herniate inferior and medial to the ICA, ${ }^{11,21}$ where the paraclinoid aneurysm of the medial type, carotid cave, and superior hypophyseal artery aneurysms occur. However, by using the base of the OS as a reference point, overestimation of intradural portion of the paraclinoid aneurysms occurred in 9 patients whose aneurysms were presumed to be intradural but turned out to be transitional, in 2 patients whose aneurysms were presumed to be intradural but turned out to be extradural, and in 1 patient whose aneurysm was presumed to be transitional but turned out to be extradural, which means the DDR is located slightly higher than the OS base (Table 3). In this series, there were a total of 6 extradural aneurysms. By using the OS base as a reference point in advance, we could detect 3 of these aneurysms preoperatively and thus could have avoided unnecessary exploration. Further improvement of the diagnostic sensitivity and specificity through the receiver operating characteristic (ROC) curve is discussed below. Of note, among these 12 patients, 10 were either the medial or posterior type. An example of overestimation is shown in Fig. 4.

\section{Predictive Value}

The ROC curve was used to determine the cut-point value for the OS as a reference point (Fig. 5). The TS had worse interrater reliability than the OS, so it was not considered for further analysis. The result for the OS was 2.050 $\mathrm{mm}$ (sensitivity 0.806 ; specificity 0.792 ), which means that the DDR was located $2 \mathrm{~mm}$ above the base of the OS in the axial planes. In other words, if the neck of the paraclinoid aneurysm traverses the axial plane $2 \mathrm{~mm}$ above (i.e., $+2 \mathrm{~mm}$ ) the base of the OS, the aneurysm is most likely transitional. If above the $+2 \mathrm{~mm}$ axial plane, the aneurysm is intradural; if completely below the $+2 \mathrm{~mm}$ plane, the aneurysm is extradural and covered by the DDR. 


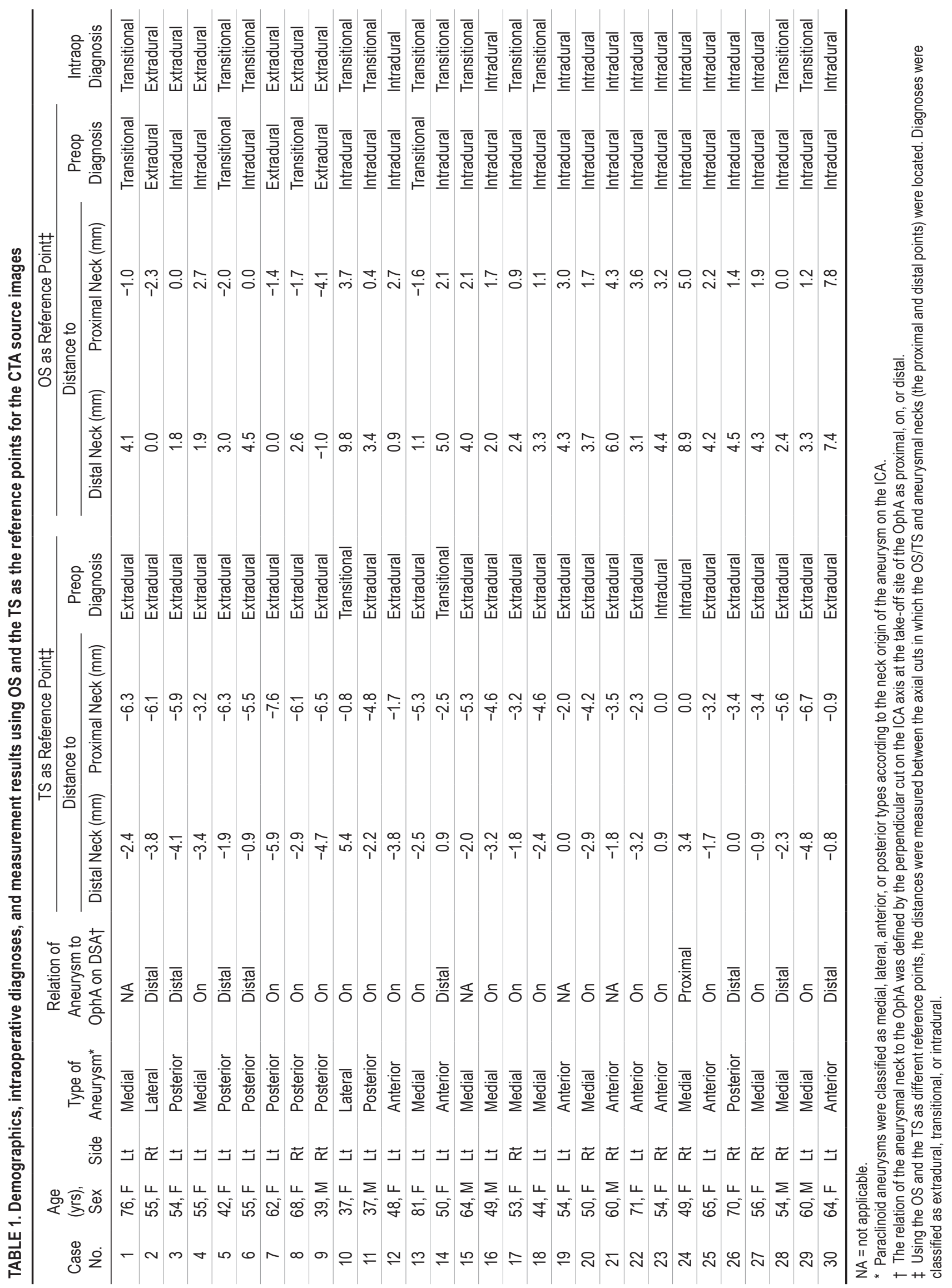


TABLE 2. The relationship between intraoperative definitive diagnosis and other factors (age, sex, laterality of aneurysm, type of aneurysm, and relation of aneurysm to OphA on DSA

\begin{tabular}{|c|c|c|c|c|}
\hline \multirow[b]{2}{*}{ Variable } & \multicolumn{3}{|c|}{ Intraop Diagnosis* $\dagger$} & \multirow{2}{*}{ - $\quad \begin{array}{c}p \\
\text { Value }\end{array}$} \\
\hline & Extradural & Transitional & Intradural & \\
\hline Sex & & & & 0.572 \\
\hline Female & 5 & 8 & 10 & \\
\hline Male & 1 & 4 & 2 & \\
\hline Laterality of aneurysm & & & & 0.179 \\
\hline Rt & 3 & 2 & 6 & \\
\hline $\mathrm{Lt}$ & 3 & 10 & 6 & \\
\hline \multicolumn{5}{|l|}{ Type of aneurysm } \\
\hline Medial & 1 & 7 & 4 & 0.011 \\
\hline Anterior & 0 & 1 & 7 & \\
\hline Lateral & 1 & 1 & 0 & \\
\hline Posterior & 4 & 3 & 1 & \\
\hline
\end{tabular}

Relation to OphA on

DSA

\begin{tabular}{lllll}
\hline Proximal & 0 & 0 & 1 & 0.673 \\
\hline On & 4 & 6 & 7 & \\
\hline Distal & 2 & 4 & 2 & \\
\hline Mean age (SD) & $56(10)$ & $54(14)$ & $58(8)$ & 0.797 \\
\hline * Values represent the number of patients unless noted otherwise. \\
$\begin{array}{l}\dagger \text { Intraoperative definitive diagnosis was only significantly correlated with the } \\
\text { type of aneurysm. }\end{array}$
\end{tabular}

\section{Discussion}

The natural history of paraclinoid aneurysms varies based on their location and anatomical relationships.
Distinguishing intradural, transitional, and extradural paraclinoid aneurysms is critical when considering treatment options. Transitional paraclinoid aneurysms-i.e., aneurysms arising from the clinoid-ophthalmic segment of the ICA and straddling across the DDR with potential risks of subarachnoid hemorrhage-necessitate treatment. ${ }^{8}$ Defining the transition of paraclinoid aneurysm from the extradural to intradural spaces can be difficult. Imaging studies that analyze the traditional anatomical landmarks, including the ACP and the origin of the OphA on DSA, ${ }^{20,22}$ have been used. Nevertheless, variability at the take-off site of the OphA on the ICA and variation in the size of the ACP make these landmarks unreliable. Recent techniques have been developed for the direct delineation of the DDR by high-resolution or contrast-enhanced 3D constructive interference MRI ${ }^{5,23}$ However, these studies either lacked direct surgical confirmation or comprised a small number of cases without analyzing diagnostic accuracy and effectiveness. In addition, MRI is more time consuming compared with CTA in emergent circumstances.

Gonzalez et al. ${ }^{3}$ demonstrated in 5 adult cadaveric heads that the inferior boundary of the OS localized the point at which the ICA pierced the oculomotor membrane (i.e., the PDR) and exited the cavernous sinus. Four surgical patients with paraclinoid aneurysms were included in their study to validate using the OS as a surrogate marker on CTA for discrimination between intradural paraclinoid $\left(\mathrm{C}_{6}\right)$ aneurysms and cavernous sinus $\left(\mathrm{C}_{4}\right)$ aneurysms. Hashimoto et al. ${ }^{4}$ analyzed the CTA images of 17 direct surgical patients between 1998 and 2005 in their hospital. They concluded that the OS was a precise identifier of the PDR, which formed the superior border of the cavernous sinus. However, the gap between the PDR and the DDR, i.e., the clinoid $\left(\mathrm{C}_{5}\right)$ segment of the ICA, was not discussed
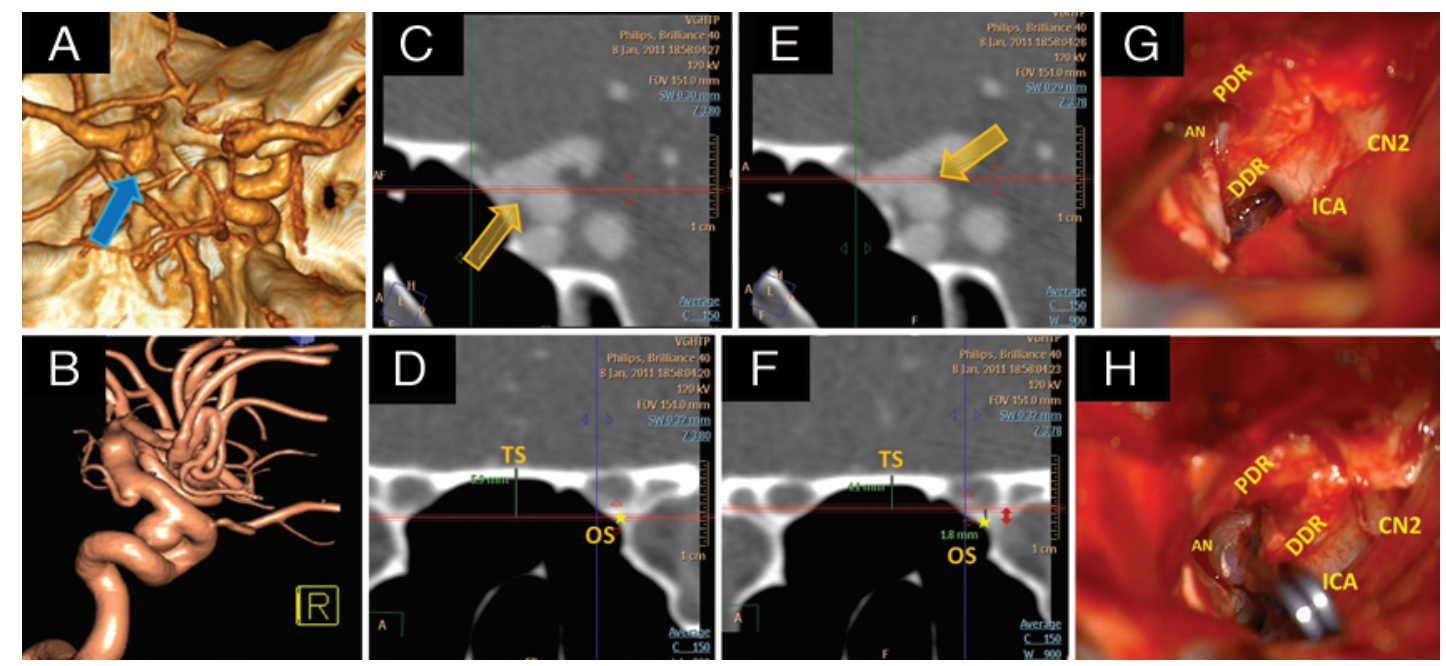

FIG. 4. Case 3. Overestimation of the intradural portion of the paraclinoid aneurysm using the OS as a reference point. A and B: CT angiogram (A) and DS angiogram (B) showing a left paraclinoid aneurysm of the posterior type. C and D: The proximal neck of the aneurysm is indicated by the yellow arrows on sagittal source CTA images. The yellow star stands for the base of the OS. Using digital cross-referencing and measurement tools, the distance between the neck of the aneurysm and the base of the OS was $0 \mathrm{~mm}$. E and F: The data of the distal neck were also retrieved in the same way, and the distance was $+1.8 \mathrm{~mm}$. The preoperative diagnosis by our definition (the base of the OS as a reference point) was an intradural paraclinoid aneurysm. $\mathbf{G}$ and $\mathrm{H}$ : The pretemporal transclinoid/transcavernous approach along with extradural anterior clinoidectomy was used for clipping. However, other than an intradural paraclinoid aneurysm, the intraoperative findings confirmed the aneurysm as extradural extracavernous. The aneurysm and its neck were found between the DDR and PDR. The aneurysm was clipped without difficulty through this approach. Figure is available in color online only. 
TABLE 3. Incidence of overestimating the intradural portion of the paraclinoid aneurysm using the $O S$ base as a reference point

\begin{tabular}{cccc}
\hline & \multicolumn{3}{c}{ Definitive Diagnosis $\dagger$} \\
\cline { 2 - 4 } Preop Diagnosis* $^{*}$ & Extradural & Transitional & Intradural \\
\hline Extradural & 3 & 0 & 0 \\
\hline Transitional & $1 \ddagger$ & 3 & 0 \\
\hline Intradural & $2 \ddagger$ & $9 \ddagger$ & 12 \\
\hline
\end{tabular}

* Preoperative diagnosis was made based on the source images from CTA with the OS base as the reference point.

$\dagger$ Intraoperative findings confirmed the definitive diagnosis.

$\ddagger$ Cases with overestimation were mostly patients harboring paraclinoid aneurysms of the medial or posterior type.

in these 2 studies. In addition, the inferior boundary of the OS ascends from the body of the sphenoid bone medially to the ACP laterally, which results in a difference in the vertical distance between the start and the end of the OS's inferior boundary. This difference causes diagnostic inaccuracy in axial cuts.

The short $\mathrm{C}_{5}$ segment of the ICA deserves our attention when dealing with paraclinoid aneurysms, and it is the DDR that serves as the boundary between intradural and extradural spaces. In this study, we decided to use an anatomical point, either the OS base or TS, to discriminate between intradural paraclinoid $\left(\mathrm{C}_{6}\right)$ and clinoid $\left(\mathrm{C}_{5}\right)$ aneurysms. To compare the diagnostic accuracy, 30 consecutive surgical cases were included. The pretemporal approach with extradural clinoidectomy allowed us to examine the relationship between the aneurysm, DDR, and PDR in an actual clinical scenario. This is the largest series to use the OS/TS as radiological landmarks for distinguishing

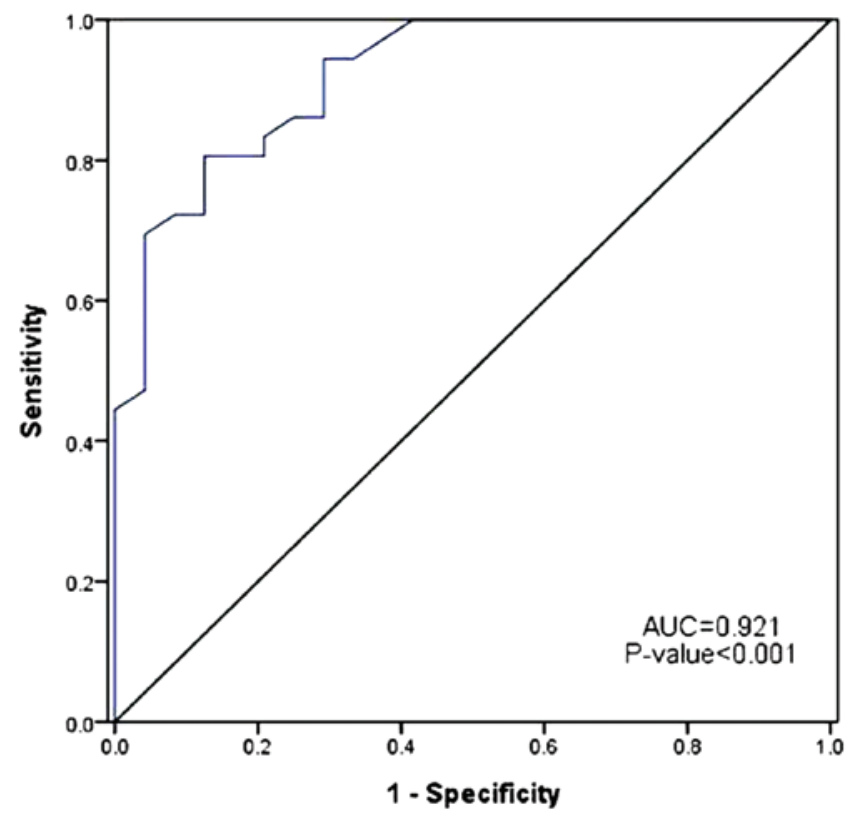

FIG. 5. ROC curve of the OS as a radiological landmark for predicting the location of the DDR. The cutoff point for the OS as a reference point is $+2.050 \mathrm{~mm}$ (sensitivity 0.806 ; specificity 0.792 ), which means that the $\mathrm{DDR}$ is located $2 \mathrm{~mm}$ above the base of the $\mathrm{OS}$ in axial planes. Figure is available in color online only. between intra- and extradural paraclinoid aneurysms with proof of direct surgical inspection. In this attempt, we supplemented with the information, which was not provided by Gonzalez et al.'s $\mathrm{s}^{3}$ and Hashimoto et al.'s ${ }^{4}$ studies, about the $\mathrm{C}_{5}$ segment of the ICA by using CTA to evaluate paraclinoid aneurysms

To measure agreement regarding the correct diagnosis, the OS is far superior to the TS on CTA. The reason is that the DDR and PDR are separated by the OS and the bulk of the ACP anteriorly but are fused at the tip of the ACP posteriorly. And, the ICA is compactly fixed together with these structures in comparison with the TS, which takes a variable shape/slope that is not related to the paraclinoid structures and the ICA. The statistics proved that the TS is not a suitable landmark for evaluating paraclinoid aneurysms.

The medial aspect of the DDR is attached more loosely and may be incomplete, allowing a small pouch of subarachnoid space to herniate inferior and medial to the ICA. ${ }^{11,21}$ This small pouch, termed the carotid cave by Kobayashi et al., ${ }^{11}$ is present in $68 \%$ to $90 \%$ of cadaveric specimens ${ }^{6,9,19}$ and is where the paraclinoid aneurysms of the medial type (or posterior type with a medial component), carotid cave, and superior hypophyseal artery aneurysms arise. Aneurysms of these types can be intradural and are at risk for subarachnoid hemorrhage. They are problematic because they project at or inferior to the ACP and can be misdiagnosed as extradural lesions on DSA. In a cadaveric study, Oikawa et al. ${ }^{19}$ observed that the DDR was located at the same level as the TS and emphasized that cave aneurysms were inferior to this plane. They did not differentiate between cave aneurysms and extradural paraclinoid aneurysms. In the current study, TS proved unreliable. We tried to avoid underestimation of intradural lesions by using the base of the OS as a reference point. However, the other way round, overestimation of intradural paraclinoid aneurysms was observed in some patients harboring paraclinoid aneurysms of the medial type (or posterior type with medial component). With the ROC curve, it is presumed that the DDR should be located $2 \mathrm{~mm}$ above the base of the OS and have good specificity and sensitivity for predicting paraclinoid aneurysms.

Instead of the usual reformatted CTA images, which are $8 \mathrm{~mm}$ thick, we chose the source images from CTA because more details could be delineated at the paraclinoid region to show the relationship between the aneurysmal neck and OS. The directions in which the aneurysms project from the $\mathrm{C}_{5}$ segment of the ICA are nearly parallel to the axial planes. Therefore, the axial source images are suitable for detecting aneurysmal neck projection at the paraclinoid region. The contour of the OS can be best identified through coronal images. Then, using the digital cross-referencing tool, the vertical distance between the axial planes of the aneurysm and the base of the OS can be calculated. Giant paraclinoid aneurysms, which may distort the anatomical features by eroding the normal osseous structures, can grow superiorly and violate the traditional anatomical boundaries. However, giant paraclinoid aneurysms rarely cause diagnostic dilemmas. Therefore, source images from CTA offer more detailed information for diagnosing paraclinoid aneurysms of medium or small sizes. 
This consecutive retrospective study contains surgical patients who underwent CTA and direct surgery. Eighty percent of the cases were intradural or transitional. A randomized, double-blind study would be ideal to confirm the effectiveness of the OS as a landmark. However, it is extremely difficult and unethical to undertake these kinds of studies because an aneurysm appearing to be obviously extradural or intracavernous would be usually observed. Methods of confirmation might be confined to retrospective studies or case studies of multiple aneurysms that are intradural, extradural extracavernous, and/or extradural intracavernous on the same side. We think the objectivity of this study was ensured because the cases were consecutive, although the number of the patients was not large, and there was a lack of balance between cases with extradural intra-/extracavernous aneurysms.

\section{Conclusions}

High-resolution thin-cut CTA is a fast and crucial tool for diagnosing paraclinoid aneurysms. The OS serves as an effective landmark in CTA source images for distinguishing between intradural and extradural paraclinoid aneurysms. The DDR is supposed to be located $2 \mathrm{~mm}$ above the base of the OS in axial planes.

\section{Acknowledgments}

We thank to the Biostatistics Task Force, Taipei Veterans General Hospital, for data processing and statistical assistance.

\section{References}

1. Bouthillier A, van Loveren HR, Keller JT: Segments of the internal carotid artery: a new classification. Neurosurgery 38:425-433, 1996

2. De Jesús O, Sekhar LN, Riedel CJ: Clinoid and paraclinoid aneurysms: surgical anatomy, operative techniques, and outcome. Surg Neurol 51:477-488, 1999

3. Gonzalez LF, Walker MT, Zabramski JM, Partovi S, Wallace RC, Spetzler RF: Distinction between paraclinoid and cavernous sinus aneurysms with computed tomographic angiography. Neurosurgery 52:1131-1139, 2003

4. Hashimoto K, Nozaki K, Hashimoto N: Optic strut as a radiographic landmark in evaluating neck location of a paraclinoid aneurysm. Neurosurgery 59:880-897, 2006

5. Hirai T, Kai Y, Morioka M, Yano S, Kitajima M, Fukuoka $\mathrm{H}$, et al: Differentiation between paraclinoid and cavernous sinus aneurysms with contrast-enhanced 3D constructive interference in steady- state MR imaging. AJNR Am J Neuroradiol 29:130-133, 2008

6. Hitotsumatsu T, Natori Y, Matsushima T, Fukui M, Tateishi J: Micro-anatomical study of the carotid cave. Acta Neurochir (Wien) 139:869-874, 1997

7. Hoh BL, Carter BS, Budzik RF, Putman CM, Ogilvy CS: Results after surgical and endovascular treatment of paraclinoid aneurysms by a combined neurovascular team. Neurosurgery 48:78-90, 2001

8. Jeon JS, Ahn JH, Huh W, Son YJ, Bang JS, Kang HS, et al: A retrospective analysis on the natural history of incidental small paraclinoid unruptured aneurysm. J Neurol Neurosurg Psychiatry 85:289-294, 2014

9. Kim JM, Romano A, Sanan A, van Loveren HR, Keller JT: Microsurgical anatomic features and nomenclature of the paraclinoid region. Neurosurgery 46:670-682, 2000

10. Kim LJ, Tariq F, Levitt M, Barber J, Ghodke B, Hallam DK, et al: Multimodality treatment of complex unruptured cav- ernous and paraclinoid aneurysms. Neurosurgery 74:51-61, 2014

11. Kobayashi S, Kyoshima K, Gibo H, Hegde SA, Takemae T, Sugita K: Carotid cave aneurysms of the internal carotid artery. J Neurosurg 70:216-221, 1989

12. Krisht AF, Hsu SPC: Paraclinoid aneurysms: Part 1: Superior (true ophthalmic) aneurysms. Contemp Neurosurg 30:1-6, 2008

13. Krisht AF, Hsu SPC: Paraclinoid aneurysms: Part II: Inferior paraclinoid. Contemp Neurosurg 30:1-6, 2008

14. Krisht AF, Hsu SPC: Paraclinoid aneurysms: Part III: Lateral aneurysms. Contemp Neurosurg 30:1-6, 2008

15. Krisht AF, Hsu SPC: Paraclinoid aneurysms: Part IV: Medial aneurysms. Contemp Neurosurg 30:1-6, 2008

16. Murayama Y, Sakurama K, Satoh K, Nagahiro S: Identification of the carotid artery dural ring by using three-dimensional computerized tomography angiography. Technical note. J Neurosurg 95:533-536, 2001

17. Ng PY, Huddle D, Gunel M, Awad IA: Intraoperative endovascular treatment as an adjunct to microsurgical clipping of paraclinoid aneurysms. J Neurosurg 93:554-560, 2000

18. Oh SY, Kim MJ, Kim BM, Lee KS, Kim BS, Shin YS: Angiographic characteristics of ruptured paraclinoid aneurysms: risk factors for rupture. Acta Neurochir (Wien) 155:14931499, 2013

19. Oikawa S, Kyoshima K, Kobayashi S: Surgical anatomy of the juxta-dural ring area. J Neurosurg 89:250-254, 1998

20. Punt J: Some observations on aneurysms of the proximal internal carotid artery. J Neurosurg 51:151-154, 1979

21. Seoane E, Rhoton AL Jr, de Oliveira E: Microsurgical anatomy of the dural collar (carotid collar) and rings around the clinoid segment of the internal carotid artery. Neurosurgery 42:869-886, 1998

22. Taptas JN: Intradural and extradural ICA. J Neurosurg 51: 877-878, 1979

23. Thines L, Lee SK, Dehdashti AR, Agid R, Willinsky RA, Wallace CM, et al: Direct imaging of the distal dural ring and paraclinoid internal carotid artery aneurysms with highresolution T2 turbo-spin echo technique at 3-T magnetic resonance imaging. Neurosurgery 64:1059-1064, 2009

24. Umansky F, Valarezo A, Elidan J: The superior wall of the cavernous sinus: a microanatomical study. J Neurosurg 81: 914-920, 1994

25. Wang Y, Li Y, Jiang C, Wu Z, Jiang F, Meng H, et al: Could the types of paraclinoid aneurysm be used as a criterion in choosing endovascular treatment? Neuro-radiologists' view. Acta Neurochir (Wien) 155:2019-2027, 2013

\section{Disclosures}

The authors report no conflict of interest concerning the materials or methods used in this study or the findings specified in this paper.

\section{Author Contributions}

Conception and design: Hsu, Liao, CJ Lin, CF Lin. Acquisition of data: Liao, Huang. Analysis and interpretation of data: Hsu, Liao, CJ Lin, Huang. Drafting the article: Liao. Critically revising the article: Hsu, CJ Lin, CF Lin, Chen, Shih. Reviewed submitted version of manuscript: Hsu, CJ Lin, CF Lin, Chen, Shih. Approved the final version of the manuscript on behalf of all authors: Hsu. Statistical analysis: CJ Lin, Huang. Administrative/ technical/material support: CJ Lin, Huang. Study supervision: Hsu, CF Lin, Chen, Shih.

\section{Correspondence}

Sanford P. C. Hsu, Department of Neurosurgery, The Neurological Institute, Taipei Veterans General Hospital, No. 201, Section 2, Shih-Pai Rd., Taipei 112, Taiwan. email: doc3339b@gmail. com. 